\title{
Playing fast and loose with mutation
}

\author{
Alison E. Mather and Simon R. Harris
}

This month's Genome Watch investigates the role of hypermutation in chronic bacterial infection and its implications for phylogenomic analyses.

Genetic variation introduced by spontaneous mutation is a major driver of bacterial evolution and adaptation. The success of new variants depends on their survival and spread through the population but, because most mutations are not beneficial, bacteria use complex DNA repair mechanisms, such as the mismatch repair (MMR) system, to reduce the accumulation of mutations. These mechanisms occasionally fail, which produces bacteria with reduced replication fidelity, so-called hypermutators. In pathogens, hypermutators often arise during chronic infection, when the ability to adapt quickly can be beneficial for evading antibiotic therapy and the host immune response. Despite the prevalence and potential importance of hypermutators, little is known about their genomic evolution.

Traditionally, hypermutators have been identified using phenotypic assays; however, whole-genome sequencing now enables more detailed examination of the phenomenon in clinical settings, as highlighted in a recent study in patients with cystic fibrosis $(\mathrm{CF})^{1}$. Marvig et al. investigated genetic changes in Pseudomonas aeruginosa isolates from patients with CF who had chronic respiratory infections. The CF lung is a constantly

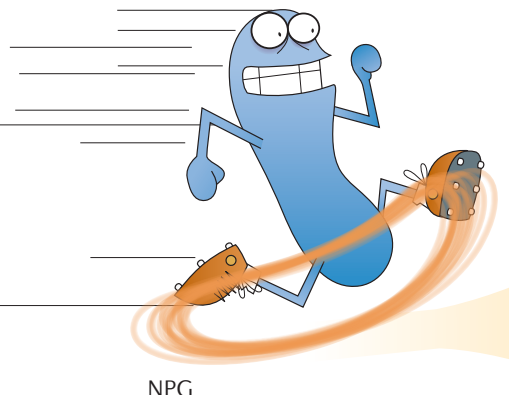

NPG changing environment, owing to tissue deterioration and repeated, prolonged antibiotic therapy. By sequencing isolates taken from patients over a 38-year period, the authors monitored how P. aeruginosa adapted to survive long-term in this environment. Hypermutator isolates, which had significantly increased numbers of single nucleotide polymorphisms (SNPs) and associated nonsynonymous mutations in MMR-related genes, were isolated from $48 \%$ of the patients. Notably, rather than being randomly spread, the genetic variations accrued by the hypermutators were more common in homopolymeric tracts, which are stretches of DNA that contain repetitions of a single nucleotide. In the $P$. aeruginosa genome, homopolymers are rare in essential genes, but are more common in genes associated with the cell envelope, modulation of which the authors hypothesize is linked to increased bacterial survival in the human host. One gene, encoding a membrane receptor protein, contained mutations in the same homopolymer in all hypermutators sequenced. This led the authors to speculate that the selective advantage of hypermutators in chronic infection might be due not only to their ability to evolve faster, but also to the preferential targeting of the mutations to specific genes.

Another study ${ }^{2}$ showed that hypermutators are not exclusive to CF-associated respiratory disease. Wang et al. found that hypermutators made up 18\% of Staphylococcus aureus isolates recovered from cows with subclinical mastitis, and there was an association between the presence of hypermutators and increased antibiotic resistance. However, hypermutation can come at a cost; for example, a hypermutating $P$. aeruginosa strain in which the MMR system was inactivated, showed decreased virulence and transmission potential in a CF mouse model ${ }^{3}$. Thus, hypermutation might be a trade-off between increased survival in the host and reduced transmissibility.

Beyond the biological consequences, hypermutation can also cause analytical problems. Evolutionary models used to estimate substitution rates and dates of historical evolutionary events assume that mutation rates are relatively stable. The inclusion of hypermutating strains in such analyses can bias results or interfere with these estimates, which can potentially lead to inaccurate conclusions. This can have implications for the growing field of genomic epidemiology in particular: using SNP thresholds to define and identify outbreaks could erroneously exclude hypermutators and miss transmission events ${ }^{4}$. Methods for including and accounting for these hypermutating strains will need to be developed on the basis of biologically-relevant criteria.

As the number of sequenced bacteria increases, our understanding of the frequency and evolutionary consequences of hypermutators will continue to increase, and will reveal the true importance of this often overlooked phenotype.

Alison E. Mather and Simon R. Harris are at the Sanger Institute, Wellcome Trust Genome Campus, Hinxton, Cambridge CB 10 1SA, UK. e-mail:microbes@sanger.ac.uk doi: 10.1038/nrmicro3164

1. Marvig, R. L. et al. Genome analysis of a transmissible lineage of Pseudomonas aeruginosa reveals pathoadaptive mutations and distinct evolutionary paths of hypermutators. PLOS Genet. 9, e1003741 (2013).

2. Wang, S. et al. Hypermutable Staphylococcus aureus strains present at high frequency in subclinical bovine mastitis isolates are associated with the development of antibiotic resistance. Vet. Microbiol. 165, 410-415 (2013).

3. Mena, A. et al. Inactivation of the mismatch repair system in Pseudomonas aeruginosa attenuates virulence but favors persistence of oropharyngeal colonization in cystic fibrosis mice. J. Bacteriol. 189, 3665-3668 (2007).

4. Köser, C. U. et al. Rapid whole-genome sequencing for investigation of a neonatal MRSA outbreak. New Engl. J. Med. 366, 2267-2275 (2012).

Competing interests statement

The authors declare no competing interests. 